\title{
Temporal perturbations in sonic hedgehog signaling elicit the spectrum of holoprosencephaly phenotypes
}

\author{
Dwight Cordero, ${ }^{1}$ Ralph Marcucio, ${ }^{1}$ Diane Hu, ${ }^{1}$ William Gaffield, ${ }^{2}$ Minal Tapadia, ${ }^{1}$ and Jill A. Helms ${ }^{1,3}$ \\ ${ }^{1}$ University of California at San Francisco, San Francisco, California, USA. ${ }^{2}$ Western Regional Research Center, \\ United States Department of Agriculture, Albany, California, USA. ${ }^{3}$ Stanford University, Stanford, California, USA.
}

\begin{abstract}
One of the most perplexing questions in clinical genetics is why patients with identical gene mutations oftentimes exhibit radically different clinical features. This inconsistency between genotype and phenotype is illustrated in the malformation spectrum of holoprosencephaly (HPE). Family members carrying identical mutations in sonic hedgehog $(\mathrm{SHH})$ can exhibit a variety of facial features ranging from cyclopia to subtle midline asymmetries. Such intrafamilial variability may arise from environmental factors acting in conjunction with gene mutations that collectively reduce SHH activity below a critical threshold. We undertook a series of experiments to test the hypothesis that modifying the activity of the SHH signaling pathway at discrete periods of embryonic development could account for the phenotypic spectrum of HPE. Exposing avian embryos to cyclopamine during critical periods of craniofacial development recreated a continuum of HPE-related defects. The craniofacial malformations included hypotelorism, midfacial hypoplasia, and facial clefting and were not the result of excessive crest cell apoptosis. Rather, they resulted from molecular reprogramming of an organizing center whose activity controls outgrowth and patterning of the mid and upper face. Collectively, these data reveal one mechanism by which the variable expressivity of a disorder such as HPE can be produced through temporal disruption of a single molecular pathway.
\end{abstract}

\section{Introduction}

Holoprosencephaly (HPE) is a congenital malformation characterized by incomplete cleavage of the embryonic forebrain (1). The clinical presentation of HPE is remarkably variable: at one end of the spectrum, HPE fetuses can exhibit cyclopia with a proboscis and a single prosencephalic vesicle while at the other extreme, obligate HPE carriers can have a normal facial appearance (2). Interposed between these phenotypic extremes are patients who exhibit varying degrees of hypotelorism, clefting, microcephaly, microphthalmia, and midfacial hypoplasia (3). The principal HPE classification scheme was based upon the degree of separation of the cerebral hemispheres (4), but more recently, molecular diagnoses have aided in identifying individuals that are either affected or obligate carriers of the disease (5). Mutations in ZIC2 (6), SIX3 (7), and transforming growth interacting factor (TGIF) (8), as well as constituents of the sonic hedgehog ( $\mathrm{SHH})$ signaling pathway (1, 9-12), are implicated in the pathogenesis of HPE.

Despite intense scrutiny, investigators have not delineated any genotype-phenotype correlations that can account for the range of HPE phenotypes (13). For example, the broad spectrum of clinical phenotypes observed in families with identical mutations in SHH (10) suggests that haploinsufficiency may be inadequate to cause HPE (14). This is further supported by the observation that some HPE patients carry mutations in both SHH and other HPE genes, such as TGIF and ZIC2 $(13,14)$.

Nonstandard abbreviations used: holoprosencephaly (HPE); 2-hydroxypropyl- $\beta$ cyclodextrin (HBC); stage (St.); terminal deoxynucleotidyl transferase (TdT); transforming growth interacting factor (TGIF)

Conflict of interest: The authors have declared that no conflict of interest exists.

Citation for this article: J. Clin. Invest. 114:485-494 (2004)

doi:10.1172/JCI200419596.
Therefore, a second insult may be required for clinical manifestation of HPE. Taken together, these genetic data and clinical observations indicate that, rather than being a single-gene disorder, HPE may be multigenic (14) and the variety of HPE phenotypes could arise from other genetic polymorphisms, additional nonallelic mutations, or environmental influences $(15,16)$. Here we explored the latter possibility, that exposure to environmental teratogens during critical periods of embryogenesis could underlie the spectrum of HPE defects.

A number of environmental factors can elicit HPE-related malformations (17). For example, preconceptual diabetes (18), retinoic acid exposure (19), maternal alcohol consumption (2022), and cholesterol synthesis inhibitors (23) can elicit HPE-like dysmorphologies. Exposure to the steroidal alkaloid cyclopamine can cause the most severe HPE phenotype of cyclopia, in addition to less severe manifestations of the syndrome such as facial clefting (24). Cyclopamine exerts its teratogenic effects by binding to the heptahelical bundle of smoothened (SMO) and thereby inhibiting SHH signal transduction (25-27). Mutations in other genes in the SHH signaling pathway also produce HPE-related defects $(1,12)$. Thus, disruptions in SHH signaling represent a common feature in the etiology of HPE.

Studies conducted in mammals and birds show that the severity of HPE defects correlates with the stage in which interruption in Shh signaling occurs $(9,28)$. Null mutations in the murine Shb gene disrupt neural plate patterning, and the affected embryos consequently exhibit cyclopia (9). Shb is expressed throughout embryogenesis and in a wide variety of tissues, but in the murine model, germline deletion of $S h b$ precludes any analyses of its later functions. Since our goal was to understand how the range of HPE phenotypes could be generated from disruptions in Shh, we turned to an avian model system. This experimental approach 

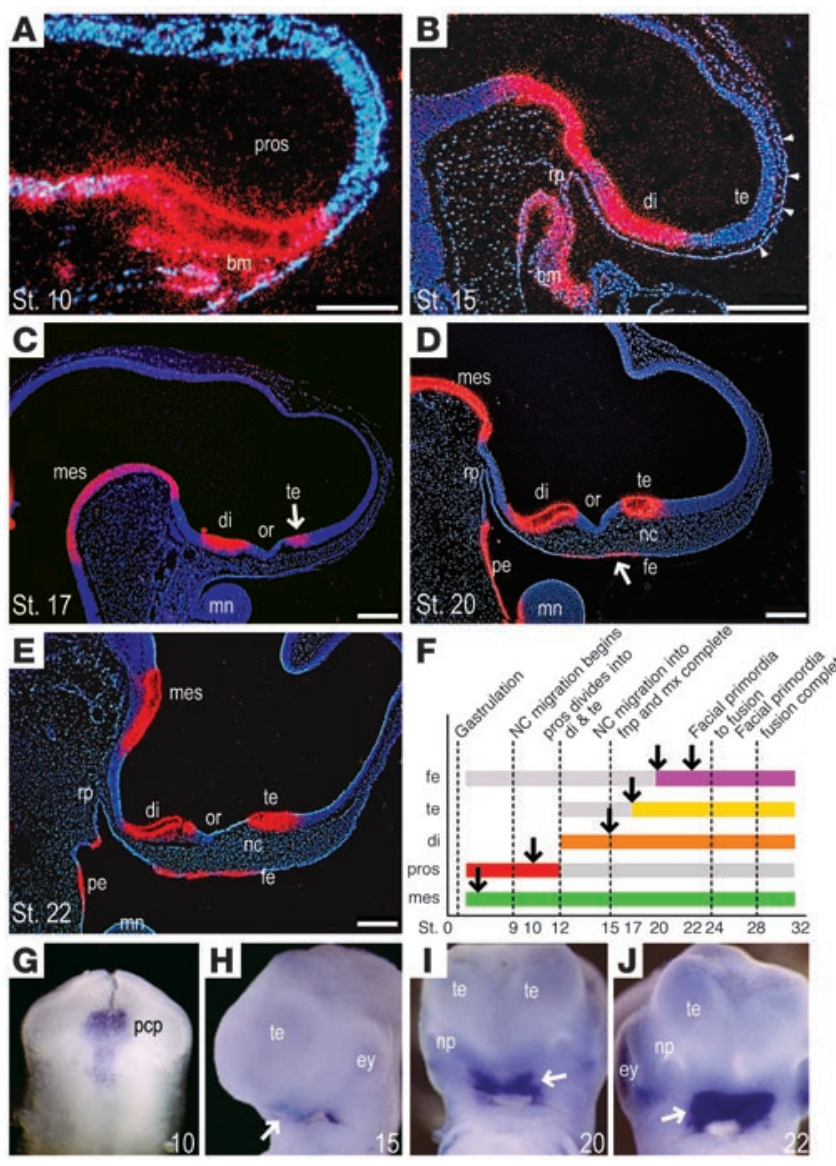

allowed us to block Shh signaling at specific stages of embryonic development and then assess whether the morphological outcomes represented the spectrum of HPE phenotypes.

\section{Results}

Shb is expressed sequentially in the brain and in the face. The first site of Shb expression in the rostral head is the midline of the neural plate (29). Following neurulation, which occurs around stage 4 (St. 4) in an avian embryo, this midline region becomes the ventral prosencephalon, where Shb continues to be expressed at St. 10 (Figure 1A). The prosencephalon subsequently divides into the telencephalon and diencephalon; at St. 15, Shb transcripts are restricted to the ventral diencephalon (Figure 1B). At St. 17, Shb is induced in the ventral telencephalon, which is separated from the diencephalic domain by the Shb-negative optic recess (Figure 1C). At St. 20, Shb is induced in ventral ectoderm of the frontonasal primordium (Figure 1D). Once established, these forebrain and facial domains of Shb persist from St. 20 onward (Figure 1E; summarized in Figure 1F). Whole-mount in situ hybridization analyses confirmed the midline Shb expression patterns and also illustrated the more lateral domains in the roof of the stomodeum (oral cavity) and in the maxillary primordia (Figure 1, G-J). These Shb expression patterns led us to speculate that the brain and the face might be differentially susceptible to Shh perturbation in a manner that recapitulated the progressive severity of the HPE phenotype. We set about testing this hypothesis by disrupting signaling at precisely those stages when $S h b$ was induced, first in the forebrain and then in the face.

\section{Figure 1}

Ontogeny of Shh expression in the craniofacial primordia. (A) At St. 10, Shh is expressed in the prosencephalon (pros) and in oral ectoderm and pharyngeal endoderm comprising the buccopharyngeal membrane (bm). (B) By St. 15 the prosencephalon has separated into the telencephalon (te) and the diencephalon (di), where Shh transcripts are localized. Neural crest cells have migrated as far as the dorsal aspect of the frontonasal primordium (arrowheads). rp, Rathke's pouch. (C) At St. 17, Shh is induced in a new domain within the ventral telencephalon (arrow). mn, mandible; mes, mesencephalon; or, optic recess. (D) At St. 20, Shh is induced in a second new domain, ventral facial ectoderm (fe, arrow), which is separated from the telencephalon by intervening frontonasal neural crest (nc). The pharyngeal endodermal (pe) domain of Shh, first evident at St. 10, persists through embryogenesis. (E) These Shh domains are preserved from St. 20 onward. (F) Illustration of the sequential induction of Shh relative to craniofacial development and to age. The time points of cyclopamine administration are indicated by arrows and correspond to the stepwise induction of Shh in the craniofacial primordia. The units of measurement on the $x$ axis correspond to Hamburger-Hamilton stages of avian development and span a period of approximately seven days. fnp, frontonasal process; $\mathrm{mx}$, maxillary process. (G) Whole-mount in situ hybridization analyses at St. 10 show Shh transcripts in the medial prechordal plate (pcp), which will contribute to the ventral neural tube after neurulation. (H) Shh transcripts in the diencephalon are barely discernible through the facial ectoderm at St. 15 (arrow). ey, eye. (I) By St. 20, Shh is strongly expressed throughout the ectoderm of the stomodeum (arrow) and the maxillary primordia (J), which persists at St. 22. Scale bars: $100 \mu \mathrm{m}$ (A); $200 \mu \mathrm{m}$ (B-E).

Cyclopamine exerts teratogenic effects in a dose- and stage-specific fashion. Cyclopamine is a well-described teratogen that causes early embryonic lethality and, in surviving embryos, elicits a range of malformations (30-35). For example, we found that embryos frequently exhibited growth restriction and kyphoscoliosis when the cyclopamine exposure occurred between St. 4 and St. 17; we later determined that these anomalies were early indicators of embryos that would develop craniofacial anomalies. The most common reproducible malformations seen outside the facial region were a reduction in tail length and abnormal morphology of the foreand hindlimbs (data not shown).

Since our primary objective was to determine whether the phenotypic spectrum of HPE could be generated from temporal disruption in Shh signaling, we had to deliver a dose of cyclopamine that reliably had a morphological effect yet did not cause excessive embryonic lethality. Most studies in the past used doses of cyclopamine that cause cyclopia and early embryonic death (28, 36). We found that when St. 4 and St. 10 embryos were used, a $5.0 \mu \mathrm{g}$ dose of cyclopamine consistently resulted in the highest proportion of live embryos with cyclopic phenotypes, but if they were exposed to even slightly higher doses, the vast majority of them died within hours (Figure 2). Furthermore, these embryos rarely survived past St. 20 even when the lower dose of cyclopamine was used.

To evaluate the effects of cyclopamine on later stages of craniofacial development we had to use slightly older embryos, since they tended to survive much longer after cyclopamine exposure. Through empirical testing we determined that exposing St. 15 and St. 17 embryos to $15.0 \mu \mathrm{g}$ cyclopamine consistently resulted in a high rate of live, affected embryos that survived to later stages of development (Figure 2). We tested higher dosages (i.e., $20.0 \mu \mathrm{g}$ and greater), but found that the $15.0 \mu \mathrm{g}$ dose showed the greatest interexperimental reproducibility and didn't kill the majority of 


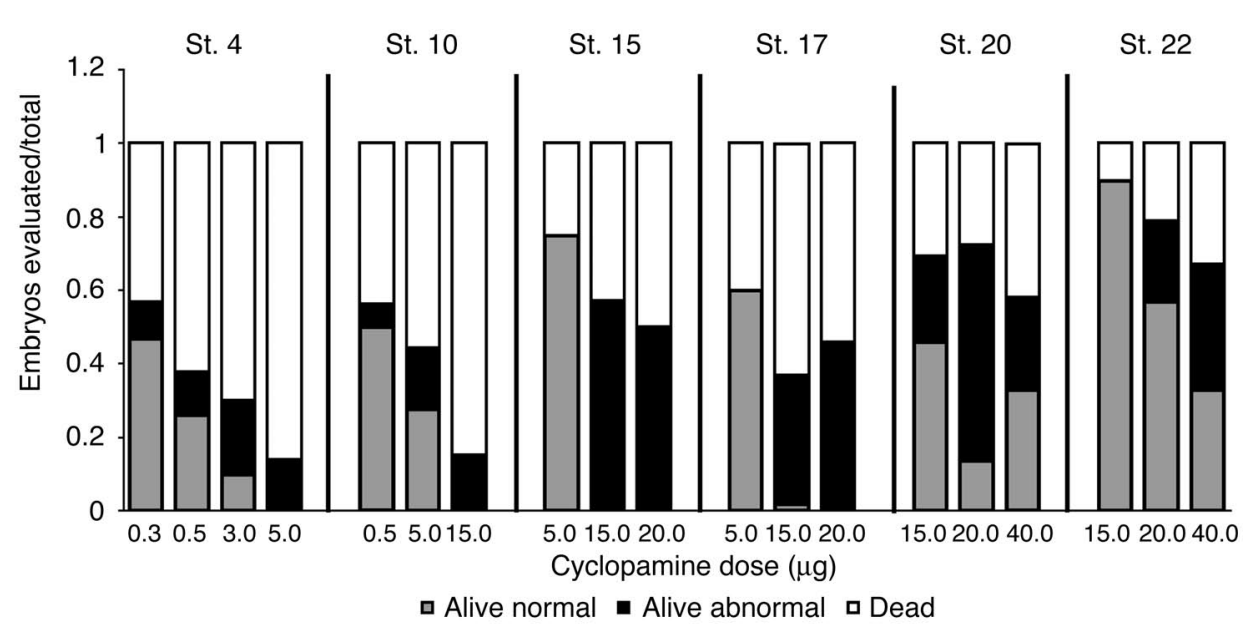

Figure 2

Dose-dependent craniofacial effects of cyclopamine. Embryos between St. 4 and St. 22 were exposed to increasing concentrations of cyclopamine ( $x$ axes) and evaluated between 3 and 72 hours after treatment to ascertain the number of living and dead embryos. Whenever feasible, embryos were also examined for evidence of gross morphological abnormalities. At this time the embryos were scored as alive and normal, alive and abnormal, or dead. The number of embryos in each of these three categories was expressed as a percentage of the total number of embryos in the cohort ( $y$ axes). The number of embryos exhibiting craniofacial malformations was directly related to the dose of cyclopamine at any given stage of embryonic development.

the embryos. For example, when St. 17 embryos were treated with $15.0 \mu \mathrm{g}$ cyclopamine, more than $95 \%$ of them exhibited the same types and severity of craniofacial malformations. Because this dose and method of delivery produced a reliable spectrum of malformations, we were able to collect embryos at earlier time points for our molecular and cellular analyses, then directly compare these data with our previous observations on the morphological consequences of cyclopamine exposure. We treated St. 20 and St. 22 embryos with $15.0 \mu \mathrm{g}$ of cyclopamine as well, and also performed a number of control experiments to show that PBS and the carrier for cyclopamine, 2-hydroxypropyl- $\beta$-cyclodextrin (HBC), did not elicit malformations on their own.

Cyclopamine exposure elicits a spectrum of craniofacial malformations. When $5.0 \mu \mathrm{g}$ of cyclopamine was delivered during patterning of the neural plate (e.g., St. 4), the vast majority of embryos died soon after exposure (Figure 2). In the few embryos we were able to collect before death, the drug elicited a cyclopic phenotype (data not shown; see also refs. 28 and 37), thus confirming the role of cyclopamine as a cyclopia-inducing teratogen. We were therefore surprised to find that no morphological abnormalities were observed when St. 22 embryos were exposed to cyclopamine, even if the dose was tripled to $15.0 \mu \mathrm{g}(n=9$; Figure 3 , A and B). These results suggested the possibility of a teratogenic threshold for cyclopamine, which was based in part on the age of the embryo.

We tested this possibility further by examining the consequences of cyclopamine exposure on St. 20 embryos. This stage of embryonic development corresponds to the time point when Shb is induced in facial ectoderm. If $15.0 \mu \mathrm{g}$ of cyclopamine was delivered at St. 20, embryos had normal or near-normal craniofacial morphology (Figure 3, C and D). A radically different outcome was observed if $15.0 \mu \mathrm{g}$ of cyclopamine was delivered just 18 hours earlier at St. 17, which corresponds to the induction of $S h b$ in telencephalic neuroectoderm. These embryos exhibited a panoply of craniofacial of Shb in the ventral midline of the neurulating embryo, caused an extreme form of hypotelorism known as synophthalmia, in which the eye fields are fused $(n=3$, Figure 3I). Taken together, these data illustrate that craniofacial tissues had a variable sensitivity to cyclopamine, which closely correlated with the onset of Shb expression in the developing brain and face.

Altering the time of Shb blockade differentially disrupts brain and facial development. Thus far we had found that by shifting the time of cyclopamine treatment we created a spectrum of craniofacial phenotypes. To better understand the relationship between the cyclopamine-induced brain and facial defects, we focused our attention on the morphological consequences of Shh signal inhibition at St. 15 and St. 17. We chose these two stages because they represent the period just before and immediately after $S h b$ induction in the telencephalon. We found that if cyclopamine exposure took place before $S h b$ was established in the telencephalon (St. 15), embryos had a single, fused telencephalic vesicle, evident both in whole-mount analyses (Figure 4A, asterisk and dotted line) and in histological sections (Figure 4B; compare with controls shown in Figure 4, C and D). Embryos also exhibited extreme hypotelorism, which resulted in the approximation of the left and right maxillary primordia (Figure $4, A$ and B, black and white arrow and the nearly fused nasal pits, red arrows). In these cases the facial defects undoubtedly arose as a consequence of the underlying structural alterations in forebrain architecture. In contrast, we found that if cyclopamine was delivered after $S h b$ was established in the forebrain but before its induction in the face (e.g., St. 17), embryos had two well-delineated telencephalic vesicles that were morphologically and histologically similar to control embryos (compare Figure 4, E and F, with Figure 4, G and H, asterisks). Despite these normal-appearing brains, however, the embryonic faces still had severe hypotelorism (Figure 4, A-D, red arrows). In these cases 

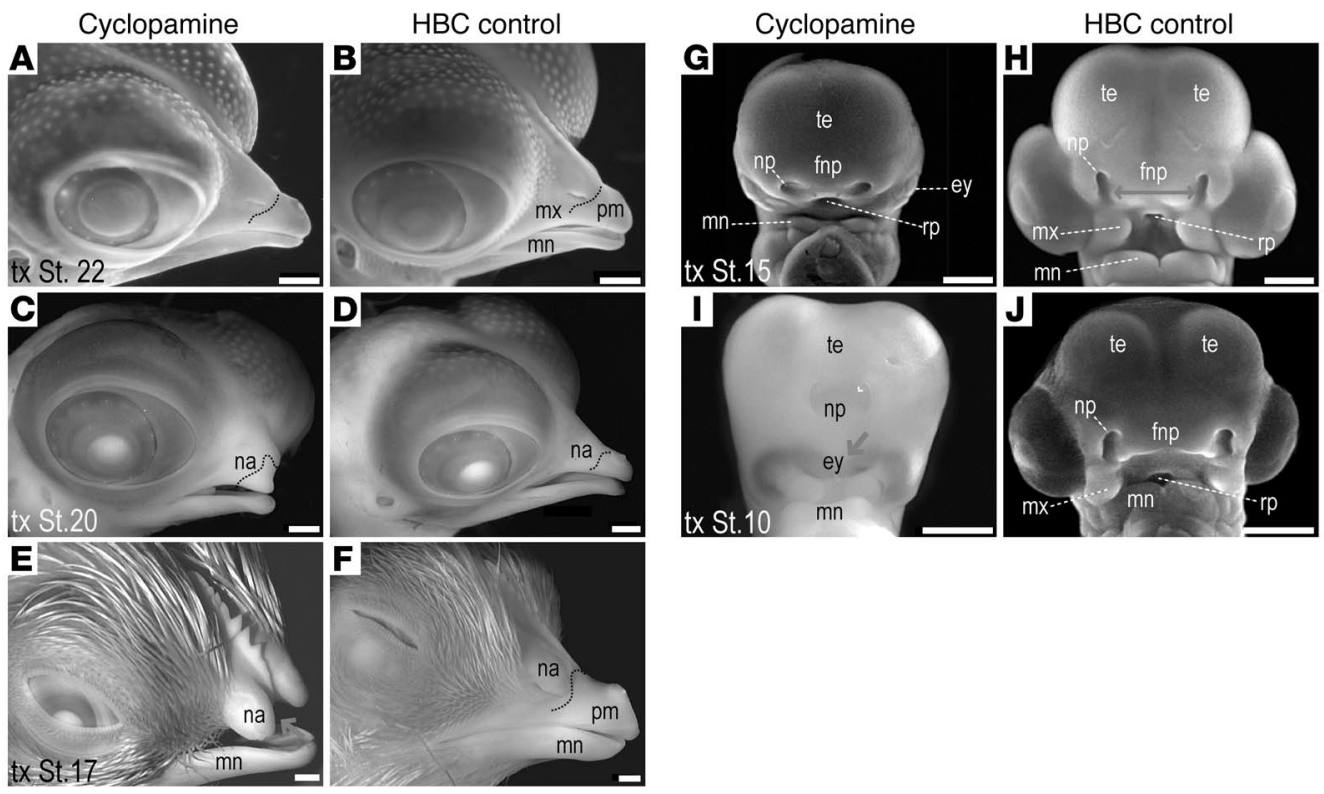

Figure 3

Cyclopamine elicits a spectrum of HPE defects. (A) Cyclopamine administration at St. 22 has no discernible effect on craniofacial development relative to (B) HBC (and untreated) controls. Note equivalent size of the premaxilla (pm) in cyclopamine and control embryos. (C) If cyclopamine is delivered at St. 20 , the premaxilla fails to extend; compare dotted line with that in (D). (E) When cyclopamine is administered at St. 17, the distal tip of the upper beak fails to form (arrow), the midface is hypoplastic, and embryos are microcephalic. Proximal facial structures and the mandible $(\mathrm{mn})$ are unaffected compared with controls $(\mathbf{F})$. (G) Cyclopamine exposure at St. 15 results in a compressed mediolateral facial axis (red arrow). Note single telencephalic (te) bulge and a severely reduced frontonasal primordium (fnp), which causes the nasal pits ( $\mathrm{np}$ ) to approximate in the midline and the eyes to deviate medially; control is shown in $\mathbf{( H )}$. The dorsoventral facial axis is also distorted, as indicated by the proximity of Rathke's pouch to the frontonasal primordium, which effectively eliminates the roof of the oral cavity. The maxillary and mandibular primordia are either absent or greatly reduced. (I) Cyclopamine delivery at St. 10 causes an arrest in midline growth, resulting in fused telencephalic vesicles and nasal pits, which create a proboscis. Midline entities such as the frontonasal primordium fail to develop compared with HBC controls $(\mathbf{J})$. The majority of embryos die within 24 hours of treatment. Scale bars: $1.0 \mathrm{~mm}(\mathbf{A}-\mathbf{F}$, I, and J); $2.0 \mathrm{~mm}(\mathbf{G}$ and $\mathbf{H})$. tx, treatment.

the hypoteloric defects could not be ascribed to an underlying brain anomaly. Instead, the hypotelorism appeared to be caused by patterning defects in the face itself.

Cyclopamine inhibits Shb signal transduction without inducing excessive neural crest apoptosis. To confirm that the cyclopamineinduced brain and facial anomalies resulted from an inhibition of Shh signal transduction, we examined embryos for changes in the expression patterns of two Shh targets, Patched (Ptc) and Gli1. Since the majority of embryos exposed at early stages died as a consequence of cyclopamine treatment (Figure 2 and ref. 28), we focused our analyses on embryos treated at St. 17. These embryos typically survive the cyclopamine exposure but still exhibit severe, reproducible craniofacial anomalies ( $n=30$; Figure $3 \mathrm{E})$. As anticipated, both Ptc and Gli1 were downregulated in response to cyclopamine (compare Figure 5, A and C with HBC controls in Figure $5, \mathrm{~B}$ and D), confirming that Shh signaling in the craniofacial tissues was repressed by cyclopamine.

Since an Shh-dependent signal is required for cranial neural crest survival (38), we reasoned that excessive cell death could account for the cyclopamine-induced craniofacial malformations. Despite exhaustive analysis, however, we detected few if any TUNEL-positive neural crest cells in the frontonasal or maxillary was not limited to facial ectoderm, since cyclopamine treatment at St. 15 blocked the induction of Shb in the telencephalon as well (data not shown). Since the mode of cyclopamine action is to inhibit the transduction of a hedgehog signal (26), these data demonstrate that Shh regulates its own expression in the frontonasal primordium and in the telencephalon.

In view of the fact that excessive neural crest cell death was not responsible for the cyclopamine-induced facial malformations, we examined the alternative possibility that cyclopamine caused some mispatterning within the frontonasal primordium. An organizing center, defined by the boundary of Shb expression in ventral facial ectoderm and Fgf8 expression in dorsal facial ectoderm, regulates proximodistal outgrowth and dorsoventral patterning within the frontonasal primordium (39). Twentyfour hours after cyclopamine exposure, Shb expression was lost and the $F g f 8$ expression domain was shifted from its dorsal location into the ventral ectodermal domain once occupied by Shbexpressing cells (compare Figure 6A with Figure 6B, arrows). At least one putative target of Fgf signaling, AP2 (40), was also shifted ventrally (Figure 6, C and D, arrows). These changes in gene expression patterns were selective, however, since the location of Barx 1 transcripts in the facial ectoderm and transcripts 
Cyclopamine

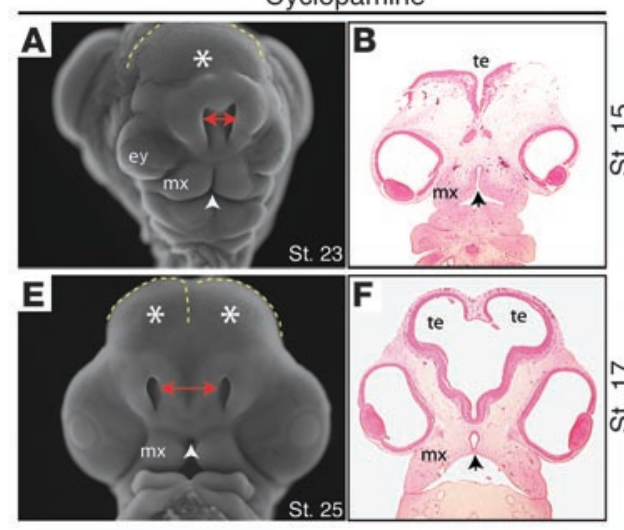

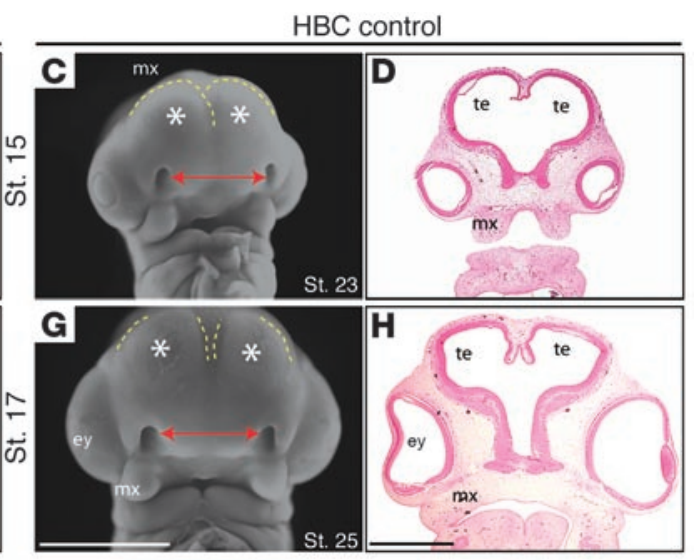

Figure 4

The teratogenic consequences of cyclopamine exposure are a reflection of the dynamic nature of Shh expression. (A and B) Whole-mount and histological assessments following cyclopamine exposure at St. 15 illustrate that both brain and facial structures are affected. Embryos exposed to cyclopamine have a single telencephalic vesicle (dotted yellow line, asterisks) and severe hypotelorism that results from a lack of expansion in the mediolateral facial axis (red arrows) and approximation of the maxillary primordia (black and white arrowheads; compare with HBC controls in $\mathbf{C}$ and $\mathbf{D}$ ). (E and F) In contrast, embryos exposed to cyclopamine at St. 17 have defects that are limited to facial structures. The telencephalon appears normal (yellow dotted lines, asterisks) compared with $\mathrm{HBC}$ controls ( $\mathbf{G}$ and $\mathbf{H})$, but treated embryos still exhibit hypotelorism (red arrows) that results in the near-approximation of the maxillary primordia (black and white arrowheads). Scale bars: $200 \mu \mathrm{m}(\mathbf{A}, \mathbf{C}, \mathbf{E}$, and G); $100 \mu \mathrm{m}$ (B, D, F, and H). mal facial morphology. Consequently, one is confronted with an apparent paradox: how can the face develop properly if the frame on which it forms is misshapen? It was this question that instigated our investigation into the link between forebrain and facial development. In this study we used the condition of HPE as a model because the forebrain and facial malformations associated with this syndrome are well-characterized and because the genetic basis for a heritable form of the disease has been identified (3).

A phenotypic spectrum of HPE defects is created by varying the timing of Shh signal blockade. A growing body of evidence indicates that HPE is a multifactorial disease whose phenotypic presentation may represent a combination of genetic and epigenetic influences $(15,16$, $43,44)$. The molecular basis for of $D l \times 2, N k x 2.1$, and $O t x 2$ in the neuroectoderm was not reproducibly altered by cyclopamine exposure (Figure 6, E-L).

Because the $S h b / F g f 8$ expression boundary is important for dorsoventral patterning in the mid and upper face (39), the absence of $S h b$ coupled with the ventral repositioning of $\mathrm{Fg} f 8$ could theoretically cause a dorsoventral patterning defect in the facial skeleton. Using whole-mount bone/cartilage staining and histological analyses of tissue sections, we found that the body of the premaxilla, which normally forms in a dorsal position at the extreme distal tip of the upper jaw, was aberrantly located ventral to the nasal capsule (compare Figure 7, A-C and G with Figure 7, D-F and H). Likewise, the nasal process of the premaxilla, which normally projects from the dorsal surface of the premaxilla, was atypically positioned on the ventral surface (compare Figure 7, $A$ and $C$ with Figure 7, D and F). Histological analyses indicated that despite its abnormal position and small size, the premaxilla maintained its articulations with the maxillary and palatine bones (Figure 7, G and H). More proximal skeletal elements were hypoplastic and foreshortened, but again, their anatomical relationships to one another were unaltered (compare Figure 7, B and G with Figure 7, E and H). Thus, perturbing Shh signaling did not eradicate distal skeletal elements in the mid and upper face but rather altered their location and size.

\section{Discussion}

A clinically ingrained edict states, "The face predicts the brain" (41). This tenet presupposes that certain types of forebrain malformations can be predicted by closely scrutinizing a patient's facial phenotype. This type of inductive reasoning is predicated on the idea that the brain serves as a scaffold upon which the face develops. Neuroimaging studies now reveal that even in the presence of abnormal forebrain morphology, patients can have a normal facial form (42). Conversely, our own data and clinical observations show that normal forebrain morphology can accompany abnor- the variable expressivity and reduced penetrance of HPE has not been identified, but one appealing hypothesis is that environmental agents act in conjunction with an autosomal dominant mutation to compromise Shh signaling. In such a scenario, one could envisage that variations in the time of teratogen exposure could elicit different phenotypes, which in turn might reflect the function of the signaling pathway during that specific developmental window. Clinical observations and our experimental evidence lend indirect support for this theory. For example, blocking Shh signaling during neural plate patterning (e.g., St. 4) causes cyclopia, while inhibiting the pathway after subdivision of the eye field (around St. 10) causes synophthalmia with a fused telencephalon (see Figure 3I). These phenotypic alterations can be ascribed to defects in midline patterning of the neural plate. If Shh signaling is blocked slightly later, then embryos still have a fused telencephalon, as well as severe hypotelorism and hypoplastic maxillae (Figure $3 \mathrm{G}$ ). In this case, the craniofacial defects are attributable to deviations in patterning the ventral forebrain region.

If Shh signaling is blocked after $S h b$ has patterned the ventral forebrain but before the gene is expressed in the face, then the phenotypic alterations are limited to the face (Figure 3E and Figure 4E). These embryos also exhibit hypotelorism, but we suspect that the basis for the hypoteloric phenotype is different between these two groups. Hypo- and hypertelorism are typically portrayed as the facial manifestation of an underlying brain anomaly. This explanation makes sense for embryos treated at St. 15, whose close-set eyes and truncated premaxillae are accompanied by a fused telencephalon. But in embryos treated at St. 17, the hypotelorism and premaxillary deficiencies are not found in conjunction with gross brain malformations. In fact, the forebrain appears to be morphologically and molecularly intact (Figure 4 and Figure 6). We propose that the malformations are due to 

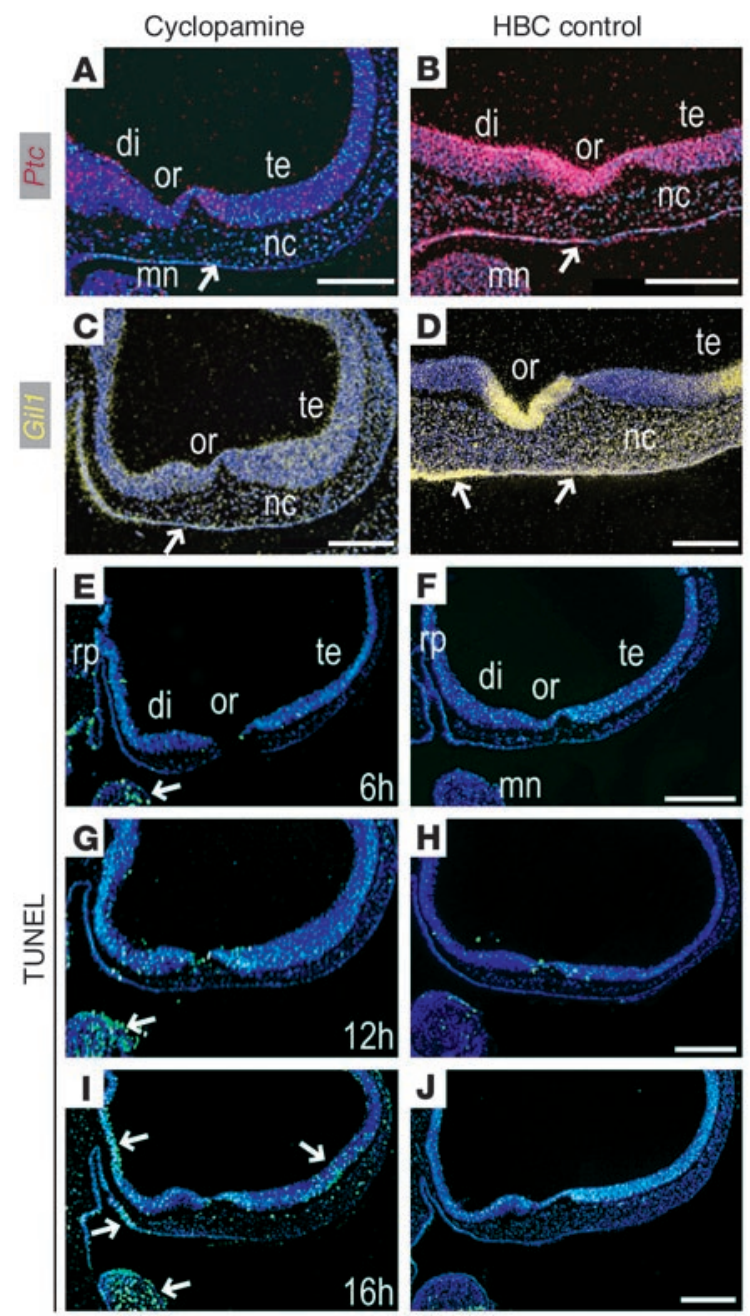

a primary defect in facial patterning rather than an abnormal brain shape. We are scrutinizing this possibility further by testing whether cyclopamine treatment specifically compromises the actual function of a frontonasal organizing center (39).

Cyclopamine is an effective inhibitor of hedgehog signaling in general, and therefore the craniofacial malformations could have arisen because of a blockade in desert hedgehog or Indian hedgehog signaling, acting either separately or in conjunction with Shh. The cyclopamine-elicited malformations shown here, however, do not phenocopy the $\mathrm{I} \mathrm{hb}^{-/-}$craniofacial defect, which is primarily restricted to ossification defects in the skull vault (45), or the $\mathrm{Dhb}^{-/-}$phenotype, which is restricted to the testis (46). Another possibility is that some craniofacial malformations may be due to disruptions in the vascular supply to the head tissues. Pericytes (47) express Ptc and they, along with endothelial cells (48), are responsive to hedgehog signaling. Consequently, craniofacial defects resulting from perturbations in Shh signaling may be further exacerbated by angiogenic defects.

The continuum of cyclopamine-induced malformations in birds created here bears a close resemblance to the spectrum of HPE phenotypes in humans, and therefore raises the possibility that epigenetic disruptions in a single molecular pathway can produce an enormous range of dysmorphologies. The extent to which these avian anomalies accurately reflect homologous

\section{Figure 5}

Cyclopamine blocks Shh signal transduction in the craniofacial complex. (A) Within 16 hours of cyclopamine exposure at St. 17, Ptc is downregulated in the diencephalic (di) and telencephalic (te) neuroectoderm, in frontonasal mesenchyme, and in facial ectoderm (arrow) relative to HBC controls (B). (C) Gli1 is similarly downregulated in the craniofacial tissues compared with HBC controls (D). (E-J) Despite these molecular changes, few cells within the frontonasal primordium undergo programmed cell death. (E) Within 6 hours of cyclopamine treatment, there is little evidence of DNA fragmentation in the craniofacial region, as indicated by TUNEL staining (arrow); compare with $\mathrm{HBC}$ control embryos (F). (G) Within 12 hours of treatment, few if any TUNEL-positive cells are detectable in the frontonasal primordium, although TUNEL staining in the mandible was increased relative to HBC-treated embryos (H). (I) Sixteen hours after treatment, TUNELpositive staining is evident in diencephalic and telencephalic neuroectoderm and in ventral facial ectoderm (arrows) compared with HBCtreated controls $(\mathrm{J})$. Scale bars: $200 \mu \mathrm{m}$.

human malformations is not entirely clear, but the conserved nature of the hedgehog signaling pathway $(49,50)$, and the fact that the arrangement of the cranial skeleton is preserved in most vertebrates (51), would suggest that the phenotypes are far more alike than they are dissimilar.

A molecular basis for the spectrum of HPE phenotypes. Cyclopamine-mediated inhibition of Shh signaling leads to a molecular mispatterning of facial ectoderm and neural crest-derived mesenchyme (Figure 6), but it remains a mystery how such changes in gene expression actually manifest as structural defects in the craniofacial architecture. One possibility is that blocking Shh signal transduction disrupts the integrity of an organizing center in the forebrain, which alters the proliferation of neural tissues $(52,53)$. Another possibility is that the cyclopamine-induced mid and upper facial defects are the consequence of massive cell death in the facial mesenchyme, as has been observed following alcohol exposure (54). Our TUNEL analyses argue against this latter etiology (Figure 4). A more probable hypothesis is that the loss of $S h b$ in the face is compensated for by the expanded function of Fgf8. In a number of developmental scenarios, Fgf's serve as survival factors (55) and thus prevent excessive cell death, at least within the first few days following teratogen exposure. This may explain why we failed to detect excessive apoptosis even after the loss of Shh signaling. In any case, the ectopic expression of Fgf8 in ventral facial ectoderm, coupled with the loss of Shh, causes a shift in the dorsoventral boundary of the frontonasal ectodermal zone, an organizing center that regulates patterned outgrowth of the frontonasal primordium (39). Other investigators have noted similar shifts in expression boundaries that result in the aberrant position of tissues. For example, ten Berge and colleagues showed that jervine exposure caused a shift in the domains of $D l x 2, A l x 3$, and $F g f 8$, which resulted in the formation of a medial incisor (56). We speculate that disruption of the frontonasal ectodermal zone adversely affects cell proliferation and/or differentiation in the chondrogenic and osteogenic condensations in the frontonasal primordium, and thus results in the aberrantly positioned and diminutive skeletal elements we observed. This remains conjecture, and studies in this area will greatly improve our understanding of how perturbations in any gene pathway materialize as structural abnormalities in a given tissue or organ.

Shb regulates its own expression in craniofacial tissues. By varying the 


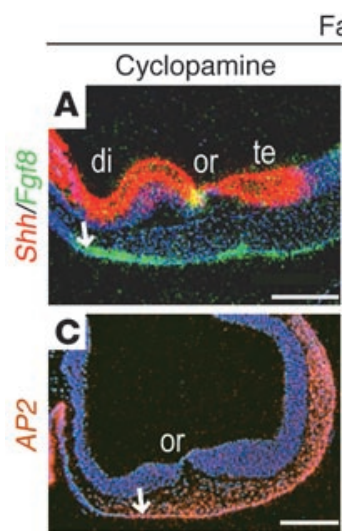

Face
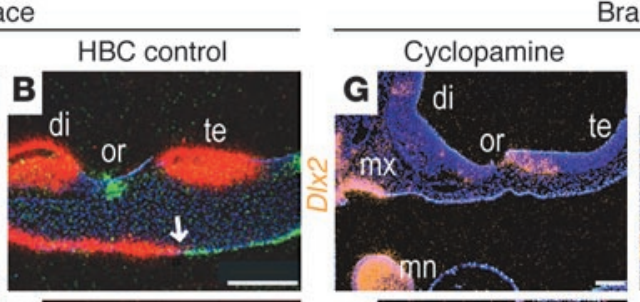

Brain
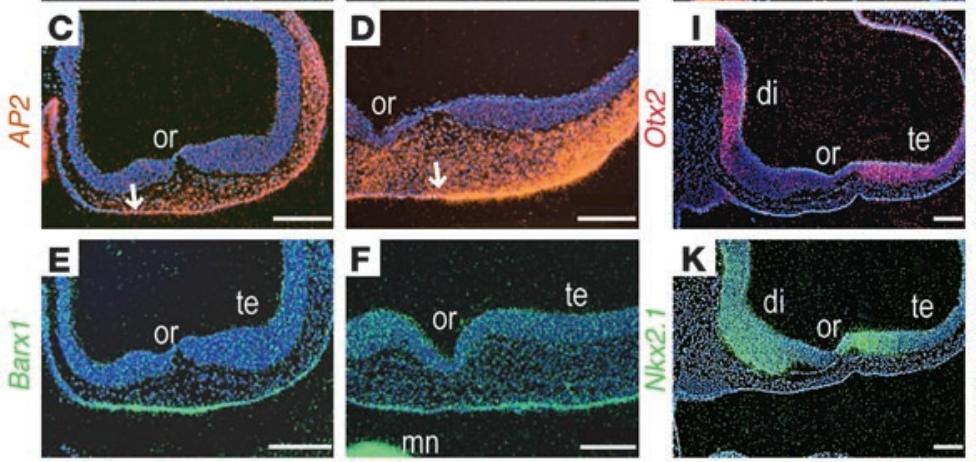

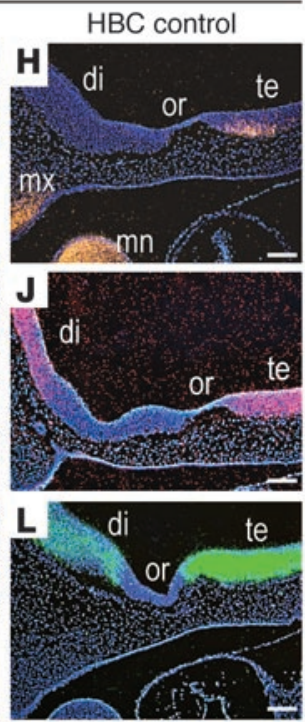

\section{Figure 6}

Cyclopamine disrupts Shh expression in facial ectoderm while leaving forebrain expression intact.

(A) Cyclopamine exposure at St. 17 blocks Shh induction in ventral facial ectoderm but does not affect its expression in the diencephalon and telencephalon. Simultaneously with the elimination of Shh, Fgf8 expands into the ventral facial ectoderm (arrow marks ventralmost extent of the domain). (B) The molecular changes described in A culminate in a loss of the Shh-Fgf8 boundary (arrow). (C) The expression domains of the transcription factor AP2 are shifted slightly in the ventral direction (arrow) compared with $\mathrm{HBC}$ controls (D), in which the ventral boundary of expression terminates distal to the optic recess (arrow). (E) The transcription factor Barx1 is not altered compared with its expression domain in controls (F). The expression domains of forebrain markers are largely unaffected by cyclopamine exposure at St. 17. (G) $D / x 2$ in the neuroectoderm of the telencephalon and diencephalon is not notably altered by cyclopamine administration relative to $\mathrm{HBC}$ controls $(\mathbf{H})$. Otx2 (I) and $N k \times 2.1(\mathbf{K})$, whose forebrain domains are indirectly regulated by Fgf8 or Shh, showed no evidence of an altered pattern of expression following cyclopamine exposure compared with HBC controls ( $\mathbf{J}$ and $\mathbf{L})$. Scale bars: $200 \mu \mathrm{m}(\mathbf{A}-\mathbf{F}) ; 100 \mu \mathrm{m}(\mathbf{G}-\mathbf{L})$.

timing of cyclopamine treatment, we found that Shh mediates its own expression within the neuroectoderm and facial ectoderm. Similar to Shh regulation in other embryonic tissues (57), this regulation likely involves at least one intermediate molecule. For example, Shb-positive neuroectoderm is in direct contact with presumptive facial ectoderm, but despite this proximity, the induction of $S h b$ in facial ectoderm does not occur for another 60 hours (Figure 1, A-D). In addition, Shb induction in frontonasal ectoderm correlates with the arrival of neural crest cells at the midline of the primordium, and previous data from our lab shows that neural crest cells can regulate Shh expression in the face (58). A third line of evidence arguing for an intermediate regulator of Shh expression in the brain and face is that exogenous recombinant Shh protein does not induce endogenous Shb expression in the face (59). This may be due to a buffering system, as has been described in the limb bud, which may limit the number of Shb-expressing cells (60). Whether an intermediate molecule that modifies Shh activity actually exists, and whether an autoregulatory feedback loop regulates Shh function in the craniofacial tissues remains unresolved and is one focus of our ongoing studies. In either case, these data provide a previously unrecognized molecular link between brain and facial development that is worthy of further exploration.

Craniofacial tissues exhibit varying sensitivity to disruptions in Shb signal transduction. This study and others $(24,30,36)$ demon-
Shb expression in pharyngeal endoderm since embryos were analyzed at multiple time points after exposure.

A more plausible explanation for the intransigent Shb domain in the pharyngeal endoderm is that newly induced domains of Shb expression are more sensitive to perturbation than those domains that are already established. When we went back and analyzed embryos with this thought in mind, we noted that Shb expression was noticeably altered only if the cyclopamine exposure occurred immediately before $S h b$ induction in a particular tissue. For example, cyclopamine completely blocked the induction of Shb expression in facial ectoderm, but only if it was delivered before establishment of the Shb domain in this region. If cyclopamine was delivered after $S h b$ induction in facial ectoderm, then there was no discernible downregulation in its expression (data not shown).

We also do not know how long teratogenic concentrations of cyclopamine exist within the embryo. The egg may act as a sink for steroidal alkaloids such as cyclopamine, and if this is the case then teratogenic levels may be present for only a brief period. We found that the expression of Ptc returned to pretreatment levels after approximately 30 hours (data not shown), which suggests that cyclopamine levels remain elevated for only a limited period. These data, however, must be interpreted in the context of qualitative rather than quantitative changes in gene expression that cannot be appreciated by in situ hybridization. Nonetheless, they do 


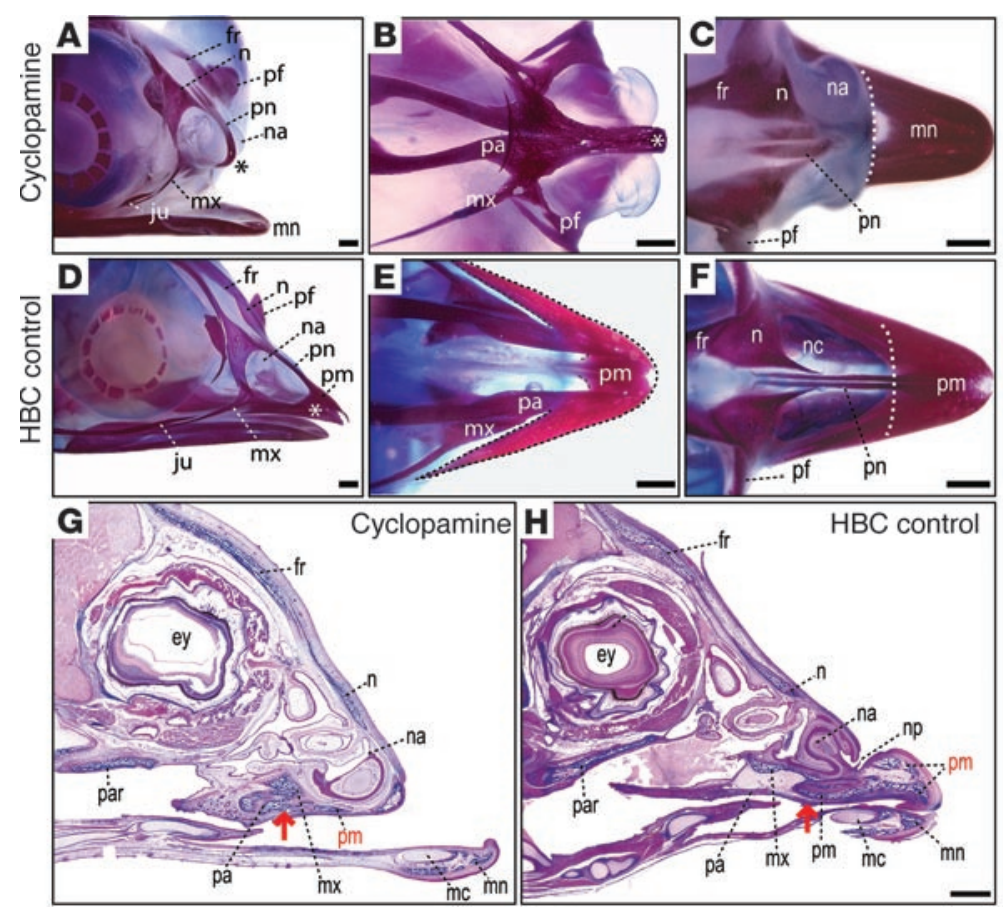

Figure 7

Loss of Shh signaling causes dorsoventral displacement of distal skeletal elements. (A) Twelve days after cyclopamine administration, distal elements of the upper beak are malpositioned and misshapen while proximal skeletal elements, including the frontal (fr), prefrontal (pf), and nasal (n) bones and the nasal capsules (na) are relatively unaffected. For example, the dorsal component of the premaxillary bone, the nasal process of the premaxilla (pn), is intact while the body of the premaxillary bone is shifted ventrally (asterisk). Skeletal elements derived from the maxillary primordia, such as the jugal (ju) and maxillary bones, are medially positioned. The mandible is unaltered in cyclopamine-treated embryos compared with $\mathrm{HBC}$ controls (D). (B) A ventral view shows that the palatine (pa), maxillary, and prefrontal bones form an atypical articulation in the ventral midline. The nasal process of the premaxilla aberrantly extends onto the ventral surface (asterisk). (C) A dorsal view reveals that the distal upper beak is truncated at the nasal capsule (dotted lines), thus the mandible becomes visible. (D) Normal craniofacial anatomy. (E) In controls, the premaxillary bone articulates with the palatine and maxillary bones. (F) A dorsal view illustrates the level of truncation (dotted line) at the premaxilla caused by cyclopamine. (G) A parasagittal section reveals that in cyclopamine-treated embryos the premaxilla is positioned ventrally and the maxillary and palatine bones are positioned medially (red arrow). (H) Control sagittal section. Scale bars: $100 \mu \mathrm{m}(\mathbf{A}-\mathbf{F})$; $1 \mathrm{~mm}(\mathbf{G}$ and $\mathbf{H})$. mc, Meckel's cartilage; par, parasphenoid.

suggest a mechanism that can explain the varying degrees of susceptibility of tissues, including those of the craniofacial complex, to the teratogenic effects of cyclopamine.

We have a very limited understanding of how mutations in one genetic pathway manifest as a range of physical deformities. In this study we explore one theory, that sequential disruption of Shh signaling in craniofacial tissues can recreate the phenotypic spectrum characteristic of HPE. By delivering cyclopamine during discrete developmental windows, we were able to identify several functions of Shh in erecting the normal architecture of the brain and face, and also separate the role of Shh in the brain from its function in the face. Our data suggest that once the molecular framework of the ventral forebrain is established, the tissue becomes relatively impervious to teratogenic disruptions. At the same time, the facial structures retain their sensi- tivity, perhaps because the facial "blueprint" has yet to be established. This model is in keeping with the observation that HPE patients can exhibit abnormal facial morphology despite normal brain architecture (62). These studies raise a number of new questions, the most pressing being to elucidate whether these mechanisms cause the phenotypic spectrum of HPE in humans.

\section{Methods}

Cyclopamine administration. Cyclopamine (11-deoxojervine) obtained from Veratrum californicum (63) was oxidized to the more potent cyclopamine-4-ene-3-one (64), and complexed with 2 -hydroxypropyl- $\beta$-cyclodextrin (Sigma-Aldrich, St. Louis, Missouri, USA). Fertilized Rhode Island Red (Gallus gallus) eggs (Petaluma Farms, Petaluma, California, USA) were incubated to stages $4,10,15,17,20$, and 22 (65), windowed to expose the embryos, and injected with PBS, HBC in PBS, or various concentrations of cyclopamine-HBC suspended in PBS. Embryos were treated with either 0.3, 0.5, 5.0, 15.0, 20.0 , or $40.0 \mu \mathrm{g}$ cyclopamine in a volume of $1.5 \mu \mathrm{l}$ by either dropping the cyclopamine on the vitelline membrane (St. 4 and St. 10) or by injecting cyclopamine beneath the vitelline membrane into the perifacial region with a glass micropipette (stages 15, 17, 20, and 22). Eggs were sealed with adhesive tape and incubated at $37.5^{\circ} \mathrm{C}$ until the desired stage of development was reached. Embryos were examined for viability and morphological changes in the craniofacial region between 3 hours and 72 hours after cyclopamine exposure. This information was used to generate the data shown in Figure 2.

In situ bybridization. Embryos were removed from the eggs, rinsed in ice-cold PBS, fixed in $4 \%$ paraformaldehyde overnight at $4^{\circ} \mathrm{C}$, and taken through a graded ethanol series to dehydration. All embryos were evaluated for phenotypic changes, following which representative embryos were subjected to in situ hybridization. Subclones of Shh, Ptc, Gli1, Fgf8, AP2, Barx1, Dlx2, Otx2, and Nkx2.1 were linearized with appropriate restriction enzymes to transcribe antisense ${ }^{35} \mathrm{~S}$ labeled riboprobes. Tissues from control and cyclopaminetreated embryos were hybridized overnight at $60^{\circ} \mathrm{C}$ with probes containing an equal number of counts per minute. Slides were washed at $60^{\circ} \mathrm{C}$ in $2 \times \mathrm{SSC}$, dipped in emulsion, and exposed for 4-6 days. Images are superimpositions of the pseudocolored hybridization signal overlaid with a nuclear stain (Hoechst 33258 dye, Sigma-Aldrich). Whole-mount in situ hybridization was performed as described (66).

Cell death assays. Untreated embryos, embryos treated with PBS and HBC (controls), and embryos treated with $15.0 \mu \mathrm{g}$ cyclopamine were analyzed for evidence of fragmented DNA using TUNEL (Roche Diagnostics Corp., Indianapolis, Indiana, USA). Tissue sections were dewaxed in Hemo-De (Fisher Scientific Co., Pittsburgh, Pennsylvania, USA), rehydrated in a graded ethanol series, treated with $10.0 \mu \mathrm{g} / \mathrm{ml}$ proteinase $\mathrm{K}$ at room temperature for 5 minutes, and rinsed in PBS. Positive control sections were treated with $3 \mathrm{U} / \mathrm{ml}$ DNase 1 (Invitrogen Corp., San Diego, California, USA). Terminal deoxynucleotidyl transferase (TdT) buffer containing fluorescein-12-2'-deoxyuridine-5'-triphosphate (Roche Diagnostics Corp.) was added to the tissue sections; negative control sections did not receive TdT. Slides were incubated at $37^{\circ} \mathrm{C}$ in the dark for 1 hour and washed with $1 \times$ PBS containing Hoechst dye. Slides were mounted in 50:50 glycerol/PBS, coverslipped, and photographed using epifluorescence optics. The images 
were merged using Adobe Photoshop (Adobe Systems Inc., Mountain View, California, USA).

Skeletal and histological analyses. For visualization of the intact cranial skeleton, whole heads were fixed in $4 \%$ paraformaldehyde and then stained with alcian blue and alizarin red, cleared in $1 \% \mathrm{KOH}$, and transferred to solutions of decreasing $\mathrm{KOH}$ strength and increasing glycerol concentrations. For histological analyses, heads were fixed as above, embedded in paraffin, sectioned, and stained using a modified Milligan's trichrome per standard protocols.

\section{Acknowledgments}

The authors gratefully acknowledge the helpful comments of members of the Helms laboratory, suggestions from S. Gross, H. Nitowsky, B. Morrow, V. Serna, and R. Pyles, and the technical assistance of M. Smith and S. Blaschko. We would also like to acknowledge the insightful critiques and comments of the anonymous reviewers. This work was supported by a T32 grant from

NIH's National Institute of Dental and Craniofacial Research (NIDCR) to D. Cordero, by NIDCR grants DE-12462, DE-00432, and DE13017 to J.A. Helms, and by the Oak Foundation.

Received for publication July 25, 2003, and accepted in revised form June 29, 2004.

Address correspondence to: Jill A. Helms, Stanford University, 257 Campus Drive, Stanford, California 94305, USA. Phone: (650) 7360919; Fax: (650) 736-4374; E-mail: jhelms@stanford.edu.

Dwight Cordero's present address is: Montefiore/Albert Einstein College of Medicine, Bronx, New York, USA.

Dwight Cordero and Ralph Marcucio contributed equally to this work.

1. Ming, J.E., et al. 2002. Mutations in PATCHED-1, the receptor for SONIC HEDGEHOG, are associated with holoprosencephaly. Hum. Genet. 110:297-301.

2. McKusick-Nathans Institute for Genetic Medicine, Johns Hopkins University (Baltimore, MD) and National Center for Biotechnology Information, National Library of Medicine (Bethesda, MD). 2000. Online Mendelian Inheritance in Man, OMIM. http://www.ncbi.nlm.nih.gov/omim/.

3. Muenke, M., and Beachy, P.A. 2000. Genetics of ventral forebrain development and holoprosencephaly. Curr. Opin. Genet. Dev. 10:262-269.

4. Gorlin, R.J., Cohen, M.M., and Levin, L.S. 1990. Syndromes of the head and neck. Oxford University Press. New York, New York, USA. 977 pp.

5. Roessler, E., and Muenke, M. 1999. The molecular genetics of holoprosencephaly: a model of brain development for the next century. Childs Nerv. Syst. 15:646-651.

6. Brown, S.A., et al. 1998. Holoprosencephaly due to mutations in ZIC2, a homologue of Drosophila odd-paired. Nat. Genet. 20:180-183.

7. Wallis, D.E., et al. 1999. Mutations in the homeodomain of the human SIX3 gene cause holoprosencephaly. Nat. Genet. 22:196-198.

8. Gripp, K.W., et al. 2000. Mutations in TGIF cause holoprosencephaly and link NODAL signalling to human neural axis determination. Nat. Genet. 25:205-208.

9. Chiang, C., et al. 1996. Cyclopia and defective axial patterning in mice lacking Sonic hedgehog gene function. Nature. 383:407-413.

10. Roessler, E., et al. 1996. Mutations in the human Sonic Hedgehog gene cause holoprosencephaly. Nat. Genet. 14:357-360.

11. Belloni, E., et al. 1996. Identification of Sonic hedgehog as a candidate gene in holoprosencephaly. Nat. Genet. 14:353-356.

12. Roessler, E., et al. 2003. Loss-of-function mutations in the human GLI2 gene are associated with pituitary anomalies and holoprosencephaly-like features. Proc. Natl. Acad. Sci. U. S. A. 100:13424-13429.

13. Nanni, L., et al. 1999. The mutational spectrum of the sonic hedgehog gene in holoprosencephaly: $\mathrm{SHH}$ mutations cause a significant proportion of autosomal dominant holoprosencephaly. Hum. Mol. Genet. 8:2479-2488.

14. Ming, J.E., and Muenke, M. 2002. Multiple hits during early embryonic development: digenic diseases and holoprosencephaly. Am. J. Hum. Genet. 71:1017-1032.

15. Dipple, K.M., and McCabe, E.R. 2000. Phenotypes of patients with "simple" Mendelian disorders are complex traits: thresholds, modifiers, and systems dynamics. Am. J. Hum. Genet. 66:1729-1735.

16. Finnell, R.H., Waes, J.G., Eudy, J.D., and Rosen- quist, T.H. 2002. Molecular basis of environmentally induced birth defects. Annu. Rev. Pharmacol. Toxicol. 42:181-208.

17. Cohen, M.M., Jr., and Shiota, K. 2002. Teratogenesis of holoprosencephaly. Am. J. Med. Genet. 109:1-15.

18. Barr, M., Jr., et al. 1983. Holoprosencephaly in infants of diabetic mothers. J. Pediatr. 102:565-568.

19. Lammer, E.J., et al. 1985. Retinoic acid embryopathy. N. Engl. J. Med. 313:837-841.

20. Bonnemann, C., and Meinecke, P. 1990. Holoprosencephaly as a possible embryonic alcohol effect: another observation. Am. J. Med. Genet. 37:431-432.

21. Ronen, G.M., and Andrews, W.L. 1991. Holoprosencephaly as a possible embryonic alcohol effect. Am. J. Med. Genet. 40:151-154.

22. Sulik, K.K., and Johnston, M.C. 1982. Embryonic origin of holoprosencephaly: interrelationship of the developing brain and face. Scan. Electron Microsc. Pt 1:309-322.

23. Edison, R., and Muenke, M. 2003. The interplay of genetic and environmental factors in craniofacial morphogenesis: holoprosencephaly and the role of cholesterol. Congenit. Anom. (Kyoto). 43:1-21.

24. Coventry, S., Kapur, R.P., and Siebert, J.R. 1998 Cyclopamine-induced holoprosencephaly and associated craniofacial malformations in the golden hamster: anatomic and molecular events. Pediatr. Dev. Pathol. 1:29-41.

25. Chen, J.K., Taipale, J., Cooper, M.K., and Beachy, P.A. 2002. Inhibition of Hedgehog signaling by direct binding of cyclopamine to Smoothened. Genes Dev. 16:2743-2748.

26. Chen, J.K., Taipale, J., Young, K.E., Maiti, T., and Beachy, P.A. 2002. Small molecule modulation of Smoothened activity. Proc. Natl. Acad. Sci. U. S. A. 99:14071-14076.

27. Taipale, J., et al. 2000. Effects of oncogenic mutations in Smoothened and Patched can be reversed by cyclopamine. Nature. 406:1005-1009.

28. Incardona, J.P., Gaffield, W., Kapur, R.P., and Roelink, H. 1998. The teratogenic Veratrum alkaloid cyclopamine inhibits sonic hedgehog signal transduction. Development. 125:3553-3562.

29. Gunhaga, L., Jessell, T.M., and Edlund, T. 2000. Sonic hedgehog signaling at gastrula stages specifies ventral telencephalic cells in the chick embryo. Development. 127:3283-3293.

30. Keeler, R.F. 1975. Teratogenic effects of cyclopamine and jervine in rats, mice and hamsters. Proc. Soc. Exp. Biol. Med. 149:302-306.

31. Omnell, M.L., Sim, F., Keeler, R.F., Harne, L.C., and Brown, K.S. 1990. Expression of Veratrum alkaloid teratogenicity in the mouse. Teratology. 42:105-119.

32. Binns, W., Shupe, J.L., Keeler, R.F., and James, L.F.
1965. Chronologic evaluation of teratogenicity in sheep fed Veratrum californicum. J. Am. Vet. Med. Assoc. 147:839-842.

33. Bryden, M.M., and Keeler, R.F. 1973. Proceedings: Effects of alkaloids of Veratrum californicum on developing embryos. J. Anat. 116:464.

34. Bryden, M.M., Perry, C., and Keeler, R.F. 1973. Effects of alkaloids of Veratrum californicum on chick embryos. Teratology. 8:19-25.

35. Keeler, R.F., and Binns, W. 1968. Teratogenic compounds of Veratrum californicum (Durand). V. Comparison of cyclopian effects of steroidal alkaloids from the plant and structurally related compounds from other sources. Teratology. 1:5-10.

36. Keeler, R.F. 1973. Teratogenic compounds of Veratrum californicum (Durand). XIV. Limb deformities produced by cyclopamine. Proc. Soc. Exp. Biol. Med. 142:1287-1291.

37. Cooper, M.K., Porter, J.A., Young, K.E., and Beachy, P.A. 1998. Teratogen-mediated inhibition of target tissue response to Shh signaling. Science. 280:1603-1607.

38. Ahlgren, S.C., and Bronner-Fraser, M. 1999. Inhibition of sonic hedgehog signaling in vivo results in craniofacial neural crest cell death. Curr. Biol. 9:1304-1314.

39. Hu, D., Marcucio, R.S., and Helms, J.A. 2003. A zone of frontonasal ectoderm regulates patterning and growth in the face. Development. 130:1749-1758.

40. Shen, H., et al. 1997. Chicken transcription factor AP-2: cloning, expression and its role in outgrowth of facial prominences and limb buds. Dev. Biol. 188:248-266.

41. DeMyer, W. 1964. The face predicts the brain: diagnostic significance of median facial anomalies for holoprosencephaly (arhinencephaly). Pediatrics. 34:256-263.

42. Cohen, M.M.J., and Sulik, K.K. 1992. Perspectives on holoprosencephaly: Part II. Central nervous system, craniofacial anatomy, syndrome commentary, diagnostic approach, and experimental studies. J. Craniofac. Genet. Dev. Biol. 12:196-244.

43. Hartman, J.L., 4th, Garvik, B., and Hartwell, L. 2001. Principles for the buffering of genetic variation. Science. 291:1001-1004.

44. Scriver, C.R. 2002. Why mutation analysis does not always predict clinical consequences: explanations in the era of genomics. J. Pediatr. 140:502-506.

45. St.-Jacques, B., Hammerschmidt, M., and McMahon, A.P. 1999. Indian hedgehog signaling regulates proliferation and differentiation of chondrocytes and is essential for bone formation. Genes Dev. 13:2072-2086.

46. Bitgood, M.J., Shen, L., and McMahon, A.P. 1996. Sertoli cell signaling by Desert hedgehog regulates the male germline. Curr. Biol. 6:298-304. 
47. Pola, R., et al. 2001. The morphogen Sonic hedgehog is an indirect angiogenic agent upregulating two families of angiogenic growth factors. Nat. Med. 7:706-711.

48. Kanda, S., et al. 2003. Sonic hedgehog induces capillary morphogenesis by endothelial cells through phosphoinositide 3-kinase. J. Biol. Chem. 278:8244-8249.

49. Hammerschmidt, M., Brook, A., and McMahon, A.P. 1997. The world according to hedgehog. Trends Genet. 13:14-21.

50. Nybakken, K., and Perrimon, N. 2002. Hedgehog signal transduction: recent findings. Curr. Opin. Genet. Dev. 12:503-511.

51. Helms, J.A., and Schneider, R.A. 2003. Cranial skeletal biology. Nature. 423:326-331.

52. Kenney, A.M., and Rowitch, D.H. 2000. Sonic hedgehog promotes $\mathrm{G}(1)$ cyclin expression and sustained cell cycle progression in mammalian neuronal precursors. Mol. Cell. Biol. 20:9055-9067.

53. Lai, K., Kaspar, B.K., Gage, F.H., and Schaffer, D.V. 2003. Sonic hedgehog regulates adult neural progenitor proliferation in vitro and in vivo. Nat. Neurosci. 6:21-27.

54. Ahlgren, S.C., Thakur, V., and Bronner-Fraser, M.
2002. Sonic hedgehog rescues cranial neural crest from cell death induced by ethanol exposure. Proc. Natl. Acad. Sci. U. S. A. 99:10476-10481.

55. Coumoul, X., and Deng, C.X. 2003. Roles of FGF receptors in mammalian development and congenital diseases. Birth Defects Res. Part C Embryo Today. 69:286-304.

56. ten Berge, D., et al. 2001. Prx1 and Prx2 are upstream regulators of sonic hedgehog and control cell proliferation during mandibular arch morphogenesis. Development. 128:2929-2938.

57. Bai, C.B., Stephen, D., and Joyner, A.L. 2004. All mouse ventral spinal cord patterning by hedgehog is Gli dependent and involves an activator function of Gli3. Dev. Cell. 6:103-115.

58. Schneider, R.A., and Helms, J.A. 2003. The cellular and molecular origins of beak morphology. Science. 299:565-568.

59. Hu, D., and Helms, J.A. 1999. The role of sonic hedgehog in normal and abnormal craniofacial morphogenesis. Development. 126:4873-4884.

60. Sanz-Ezquerro, J.J., and Tickle, C. 2000. Autoregulation of Shh expression and Shh induction of cell death suggest a mechanism for modulating polarising activity during chick limb development.
Development. 127:4811-4823.

61. Couly, G., Creuzet, S., Bennaceur, S., Vincent, C., and Le Douarin, N.M. 2002. Interactions between Hoxnegative cephalic neural crest cells and the foregut endoderm in patterning the facial skeleton in the vertebrate head. Development. 129:1061-1073.

62. Muenke, M., and Cohen, M.M., Jr. 2000. Genetic approaches to understanding brain development: holoprosencephaly as a model. Ment. Retard. Dev. Disabil. Res. Rev. 6:15-21.

63. Gaffield, W., Benson, M., Lundin, R.E., and Keeler, R.F. 1986. Carbon-13 and proton nuclear magnetic resonance spectra of Veratrum alkaloids. J. Nat. Prod. 49:286-292.

64. Gaffield, W., Incardona, J.P., Kapur, R.P., and Roelink, H. 1999. A looking glass perspective: thalidomide and cyclopamine. Cell. Mol. Biol. 45:579-588.

65. Hamburger, V., and Hamilton, H.L. 1951. A series of normal stages in the development of the chick embryo. J. Morphol. 88:49-92.

66. Albrecht, U.E.G., Helms, J.A., and Lin, H. 1997. Visualization of gene expression patterns by in situ hybridization. In Molecular and cellular methods in developmental toxicology. G.P. Daston, editor. CRC Press. Boca Raton, Florida, USA. 23-48. 